\title{
A systematic review of treatments for Impulse Control Disorders and related behaviours in Parkinson's Disease
}

\author{
Word count: 4,536 (excluding Table)
}

Puja Tanwani ${ }^{\text {a }}$, Bruce A. Fernie ${ }^{\mathrm{a}, \mathrm{b}}$, Ana V. Nikčević ${ }^{\mathrm{c}}$, Marcantonio M. Spada ${ }^{\mathrm{d}, *}$

\footnotetext{
${ }^{a}$ King's College London, Institute of Psychiatry, Department of Psychology, London, UK

${ }^{\mathrm{b}}$ CASCAID, South London \& Maudsley NHS Foundation Trust, London, UK

${ }^{\mathrm{c}}$ Kingston University, Kingston upon Thames, UK

${ }^{\mathrm{d}}$ London South Bank University, London, UK

${ }^{*}$ Correspondence to; Department of Psychology, School of Applied Science, London South Bank University, United Kingdom. Tel. +44 (0)20 7815 5760, e-mail spadam@lsbu.ac.uk.
}

October 2014

Revision 2

(c) 2016, Elsevier. Licensed under the Creative Commons Attribution-NonCommercial-

NoDerivatives 4.0 International http://creativecommons.org/licenses/by-nc-nd/4.0/ 


\begin{abstract}
Impulse Control Disorders (ICDs) are a set of behaviours characterised by impulsivity despite known harm. Related to ICDs is the dopamine dysregulation syndrome (DDS), which is characterised by an addiction-like consumption of dopaminergic medication and punding. These behaviours all have an increased prevalence in Parkinson's disease (PD). The aim of this review is to identify treatments available for patients suffering from ICDs, DDS and punding in PD. Searches of The Cochrane Controlled Trials Register, Embase, Medline and PsychInfo were conducted, using the entire timescale available. Seven out of the 688 papers retrieved met the inclusion criteria and were considered in this systematic review. One class I study, one class II study, and five class IV studies were identified. All studies demonstrated a positive effect on ICDs in PD. Research in this field is still in its early stages. At present, there is insufficient evidence to recommend any treatment over another. There is a need for more methodologically robust research, using larger, more generalizable samples, randomisation and meaningful follow-up periods. Additionally, the use of a validated outcome measures should be implemented in future research efforts.
\end{abstract}

Key words: Impulse Control Disorders; Impulse-Compulsive Behaviours; Parkinson’s Disease; Systematic review; Treatment. 


\section{Introduction}

Parkinson's disease (PD) is primarily a neurodegenerative disorder characterised by a loss of dopaminergic neurons in the nigrostriatal pathway, resulting in a classic repertoire of motor symptoms: i.e. bradykinesia, resting tremor, rigidity, and postural instability. These motor symptoms have long been the hallmark of both diagnosing and treating PD with treatment aimed at providing symptomatic relief by attempting to replace the lost dopamine. This is predominantly managed either with dopamine agonists or by using Levodopa, a dopamine precursor. Neurodegeneration of the nigrostriatal contributes to both motor and non-motor symptoms (e.g. sleep disorders, autonomic dysfunction, and neuro-endocrinal problems; Chaudhuri and Schapira, 2009; Politis et al., 2008). These symptoms can be broadly classified into neuropsychiatric problems (including disturbances in cognition and mood), sleep disturbances and autonomic symptoms. These non-motor symptoms commonly dominate the clinical picture in severe PD and, as such, have a significant impact on quality of life and disability (Rahman et al., 2008). The pathophysiology of these symptoms remains poorly understood. This, coupled with the emphasis placed on the motor symptomatology of $\mathrm{PD}$, has led to the non-motor complications gaining a relatively late entry into the field of treatment research. Notwithstanding this, in the past few decades a greater emphasis has been placed on non-motor symptoms with new treatment avenues being identified (Wood et al., 2010).

\subsection{Impulse-Compulsive Behaviours}

For the purpose of this review, Impulsive-Compulsive Behaviours (ICBs) will be used as an umbrella term to include Impulse Control Disorders (ICDs), Dopamine Dysregulation Syndrome (DDS) and punding. ICDs are defined as a set of behavioural disorders characterised by repetitive, maladaptive, and disinhibited behaviours that an individual engages in despite being aware of their potentially harmful consequences to the self or others 
(Association, 2000). The most common ICDs in PD include pathological gambling (PG), hypersexuality (HS), compulsive eating (CE), compulsive buying (CB), kleptomania, trichotillomania (repetitive hair pulling), intermittent explosive behaviour (recurrent outbursts of aggression), and pyromania (deliberate fire-setting). DDS is characterised by a compulsive use of dopaminergic medication, in a similar manner to substance addiction (Giovannoni et al., 2000), dopaminergic medication hoarding, continual self-medication despite the onset of severe dyskinesia, and hypomania (Giovannoni et al., 2000). Punding is defined as a compulsive, repetitive, and purposeless behaviour (which the patient recognises as being meaningless), usually causing isolation, social withdrawal, and irritation for the individual when the behaviour is interrupted (Evans et al., 2004).

ICBs are common neuropsychiatric complications seen in PD. The DOMINION study, an epidemiological study looking at the incidence of ICBs in PD across movement disorder centres in USA and Canada, reported a prevalence of $13.6 \%$ compared to $1 \%$ in the general population (Ferris et al., 1996; Weintraub et al., 2010a). However, the actual incidence may be higher as a result of underreporting due to the stigma and shame attached to many of these behaviours (Weintraub et al., 2006).

\subsection{Pathophysiology}

The mechanism underlying the development of these behaviours has not been fully elucidated, although the use of dopamine agonists has been identified as a risk factor (Voon et al., 2006; Weintraub et al., 2010a) with studies showing the reversal of symptoms on dopamine agonist discontinuation (Mamikonyan et al., 2008). Additionally, several papers have identified associations between ICBs and polymorphisms of genes involved in metabolising dopamine and dopamine receptors, indicating a genetic predisposition to ICBs (Cormier et al., 2013; Eisenegger et al., 2010). Other risk factors for ICBs in PD include being male, developing PD at a younger age, having a personal or family history of addictive 
behaviour, experiencing depressive symptoms, and having a novelty seeking personality (Pontone et al., 2006; Voon et al., 2006; Weintraub, 2009).

Despite the pathophysiology of ICBs in PD being unclear, the mesolimbic pathway of the brain has been implicated in its development (Cilia et al., 2008), along with the ventral striatum and prefrontal cortex, where alterations in the responsiveness to reward and punishment are thought to occur (Reuter et al., 2005).

More recently, a bio-psycho-social model of ICBs has been proposed which purports that impulsive behaviours arise from maladaptive coping mechanisms to deal with the psychological distress of dealing with a chronic condition, especially in a younger population where the disability has a greater impact on quality of life (Delaney et al., 2012). In the proposed model, psychological distress predisposes patients to developing ICBs and dopamine agonists act to multiply the susceptibility to ICBs. If this is above a certain threshold, ICBs arise, offering an explanation as to why only a subset of patients are affected (Delaney et al., 2012).

To date, no evidence-based method has been established for treating ICBs in PD. Management typically consists of dose reduction or discontinuation of dopamine agonists, which can be coupled with an increase in levodopa dose (Mamikonyan et al., 2008), however, there are several limitations to this approach. Firstly, care must be taken to balance ameliorating the ICB symptoms with preventing an increase in motor symptoms. Dopamine agonists have been established as an effective treatment for the motor symptoms of PD (Stowe et al., 2008) and therefore staying on the treatment takes precedent over a nonevidence based method of tackling ICBs. Secondly, abrupt cessation or dose reduction of dopamine agonists can lead to the development of a dopamine agonist withdrawal syndrome (DAWS; Limotai et al., 2012). DAWS is defined as a group of symptoms, both physiological and psychological, resembling those of other drug withdrawal syndromes. Symptoms such as 
dysphoria, anxiety, and drug cravings are present and have been shown to occur in a drugdependent manner (Pondal et al., 2013). Additionally, the cravings present can lead to hesitation on the patient's behalf in stopping treatment despite the ongoing ICBs and dyskinesia present with excessive use (Rabinak and Nirenberg, 2010). Furthermore, despite treatment reduction and switching to Levodopa, some patients remain drug refractory and ICBs prevail (Ávila et al., 2011; Bermejo, 2008a; Kurlan, 2004), indeed levodopa has been identified as a causatory agent (Ávila et al., 2011). The significant impact of ICBs on a patient's quality of life gives rise to the need for treatment without these limitations (Leroi et al., 2011; Nikitina et al., 2013). The aim of this review is to track progress towards an evidence-based treatment for ICBs in PD and to identify future research needs.

\section{Methods}

This systematic review was conducted at Institute of Psychiatry, King’s College London, UK using studies retrieved by performing electronic searches of the Cochrane controlled trials register, MEDLINE, EMBASE, and PsychInfo, along with the references of identified papers. The entire timescale was used up to February 2014 (week 8) inclusive.

The search strategy employed the following keywords: "Parkinson’s Disease” and “Impulse Control Disorders” or “Dopamine Dysregulation Syndrome” or “DDS” or “punding”, in combination with “management” or "treatment” or "therapeutics” or "CBT” or “pharmacological therapy” or “drug therapy” or "pharmacological interventions”. The inclusion criteria were studies: (1) investigating at the effectiveness of any type of treatment on for impulse control disorders in PD; (2) with participants presenting a clinical diagnosis of idiopathic PD currently on medication for PD, including all genders and ages; (3) with participants with no co-morbidities such as dementia (MMSE $>24$ ), psychosis, or any other neuropsychiatric complications; (4) assessing treatment according to DSM-IV criteria (American Psychiatric Association, 1994) or using a validated outcome measure; and (5) 
utilising all methodological designs (where full-text was available). The exclusion criteria were studies carried out on animals, not available in English, or with retrospective data analysis (i.e. studies needed to be testing an active treatment with the aim of reducing ICBs).

Initially titles were screened and duplicates were excluded. The remaining abstracts were screened and studies were excluded using the inclusion/exclusion criteria. After the first screening, the remaining full text articles were assessed against a quality checklist in consideration of selection, performance, detection, attrition and reporting biases. The following details were collected from each study: bibliographic details (i.e. author, country, and date of study); design of study; number of participants; mean participant age; percentage of male patients; method of assessing ICBs; type of treatment; outcome measures; average follow-up period; methods of statistical analysis; findings and conclusions of authors (see Table 1).

\section{Results}

Six hundred and eighty eight studies were retrieved through the search strategy. Of these, 124 were duplicates and 530 were excluded on abstract review because inclusion/exclusion criteria [studies done on animals (12), not in English (six), not dealing with the clinical condition (178), not looking at treatment of the clinical condition (162) and literature reviews (171)]. 34 full-text studies were read and further assessed for eligibility (inclusion/exclusion criteria). These were also subjected to a quality checklist (described above). Twenty seven studies were excluded (10 based on the exclusion criteria and 17 as a result of the quality checklist). Seven studies were included in this systematic review.

\subsection{Pharmacological Treatment}

One class I and five class IV trials were identified that evaluated pharmacological treatments for ICBs. All the pharmacological agents reviewed demonstrated positive effects on reduction of ICBs. The class I trial was a double blind RCT looking at the effect of 200mg/day 
Amantadine against placebo control lasting 17 weeks and included 17 patients with pathological gambling (Thomas et al., 2010). A combination crossover and open label design was used, with patients switching from intervention to control group (and vice versa) after two weeks of treatment (with a one week washout period in between) before a final stage where all patients were put on active treatment for two weeks. All patients showed a reduction in pathological gambling behaviour, measured using the Yale-Brown Obsessive Compulsive Scale (Y-BOCS; Goodman et al., 1989) and the Gambling Symptom Assessment Scale (G-SAS; Kim et al., 2009).

Emilio Bermejo et al. (2010) reported positive effects of 200mg/day of Zonisamide (an anticonvulsant) on ICBs, assessed using the Barratt Impulsiveness Scale (BIS; Patton and Stanford, 1995) and the Clinical Global Impression (CGI; Guy, 1976). A decrease in both outcome measures was reported (statistical significance was not reported) with a marginal change in motor symptoms. Adverse events were reported in four patients (26\%) and one patient dropped out due to lack of effectiveness.

The remaining studies were small case reports $(\mathrm{n} \leq 4)$ and looked at various drugs: Finasteride (Bortolato et al., 2012), Naltrexone (Bosco et al., 2012), Clozapine (Hardwick et al., 2013) and Valproate (Sriram et al., 2013). All the case reports demonstrated an improvement in ICB symptomatology using a variety of outcome measures.

\subsection{Psycho-social Treatment}

The single class II trial that used a psycho-social treatment for the management of ICDs in PD identified was a RCT that featured 45 patients (Okai et al., 2013). Twenty-eight patients were assigned to an intervention group that consisted of 12 sessions of nurse-led CognitiveBehavioural Therapy (CBT) and standard medical care, comprising of psycho-education (e.g. the provision of leaflets about treatment and adverse effects) and continual review by the multi-disciplinary team. The control group received standard medical care only. ICBs were 
initially screened for using the Questionnaire for Impulsive-Compulsive Disorders in Parkinson's disease (QUIP; Weintraub et al., 2009b), and diagnoses subsequently made following a clinical interview using the DSM-IV criteria for ICDs (American Psychiatric Association, 1994). Results revealed a statistically significant decrease in ICBs $(p=0.002)$ and a decrease in overall severity of symptoms $(p=0.004)$ following the CBT intervention, which were assessed using the Impulse Control Behaviour Severity Scale (ICBSS; developed by the study authors) and CGI respectively.

\section{Discussion}

All treatments included in this review demonstrated an amelioration of ICB symptomatology but research is nascent. Good quality studies looking at the treatment of ICBs in PD are scarce, especially those evaluating CBT. Case studies are rich in descriptive data but can be subject to bias (particularly selection bias), which significantly decreases the generalizability of results.

Although the results from the RCT looking at CBT were positive, care must be taken with its interpretation. For example, the study utilised a validated measure for screening for ICBs but a study-author generated tool to assess the effect of treatment, for which little data exists to assess its psychometric properties. Furthermore, there was a relatively high dropout rate in the study, with only $58 \%$ of patients in the intervention group completing all sessions. No adverse events were reported, however no reason was given for the high dropout rate and no intention-to-treat analysis was conducted either. A post-hoc analysis would be useful adjunct to such studies to assess reasons for drop-out because CBT is a promising avenue to explore, especially because it does not risk interaction effects with Levodopa or dopamine agonists, that is present with pharmacological treatments for ICBs. One reason for the low compliance could be due to mobility issues in $\mathrm{PD}$, especially severe $\mathrm{PD}$, so options looking at alternative delivery for psychosocial treatment could potentially be pursued. 
Amantadine is an NMDA receptor antagonist which is currently used in PD to decrease the dyskinesia produced by Levodopa (Luginger et al., 2000). The trial looking at amantadine demonstrated a statistically significant decrease in ICBs and was methodologically sound. However, it had a very short follow-up period (4 weeks) and, similarly to the CBT trial, had a high dropout rate (29\%). This was due to adverse events, notably reports of hallucinations and confusion. Additionally, the effects were very shortlived and consequently, in the washout period, an increase in gambling scores was seen after just one week.

A post-hoc analysis of a large multi-centred epidemiological study showed a significant association between amantadine use and ICBs (17.6\% of patients on amantadine vs. $12.4 \%$ of those not on amantadine) (Weintraub et al., 2010b). More studies are necessary to determine the exact effect amantadine has on ICBs because it is already used in PD and is known to have a beneficial effect on motor symptomatology. Future studies should also determine whether the side effects of amantadine are tolerable in this already debilitated population.

Zonisamide (a sulphonamide anticonvulsant) and Naltrexone (an opioid receptor antagonist), Clozapine (an antipsychotic) and Valproate were all effective at treating ICBs, However, all the studies evaluating these pharmaceutical agents have been small and without controls. Larger studies with higher methodological qualities are needed to more thoroughly establish efficacy. A large-scale double-blind RCT has been conducted that investigates the effect of naltrexone on ICBs in PD, however it has not yet been published so the results are unavailable (Weintraub, 2013).

The range of ICBs (ICDs, DDS, punding, etc.) in PD makes selection of a validated outcome that can assess the associated behaviours problematic. For some ICDs, like PG, specific and validated outcomes measures are available, whereas for the less common ICDs 
and DDS the authors could not identify a disorder-specific validated scale. One study resorted to using non-specific measures like the CGI in combination with the BIS (Bermejo, 2008b), which was not designed for PD population. However, the QUIP has been shown to have both high sensitivity and selectivity with regards to ICBs (regardless of whether it is completed by the patient or a carer) and a rating scale (QUIP-RS) has just been devised (Weintraub et al., 2009a; Weintraub and Siderowf, 2012). An advantage of the QUIP is that it encompasses ICDs, DDS, and punding, and has been developed specifically for patients with PD. Although it was created in 2009, none of the studies reviewed here used it as an outcome measure.

Deep Brain Stimulation (DBS) has also been implicated in ICBs in PD, with some suggestion that DBS may induce ICBs (Halbig et al., 2009). However, because patients who have undergone DBS can reduce their dopaminergic medication, DBS can lead to a reduction in ICBs (Demetriades et al., 2011). A recent review (Demetriades et al., 2011) identified four studies and 37 case reports investigating the role of DBS in ICBs. The review concluded that the nature of the relationship between DBS and ICBs is unclear. None of the studies that were identified in this review met our inclusion/exclusion criteria because the primary intention of DBS was not to reduce ICBs.

Currently there is insufficient evidence to support or refute the effectiveness of the aforementioned treatments in improving ICBs in PD. The research is still in its early stages and although positive results have been obtained for all the studies included in this review, the studies are generally small and lacking controls. Furthermore, only one study has been carried out for each treatment. However, these studies act as stepping-stones to direct future research. The use of a standardised outcome measure should be implemented when conducting research in this field. Questionnaires such as the QUIP or QUIP-RS could be used, which have been specifically designed for ICBs in PD and have been shown to have high sensitivity for the detection of ICBs in this population. 


\section{Acknowledgements}

Author BAF receives salary support from the National Institute for Health Research (NIHR) Mental Health Biomedical Research Centre and Dementia Research Unit at South London and Maudsley NHS Foundation Trust and King’s College London. The views expressed are those of the author and not necessarily those of the NHS, the NIHR or the Department of Health.

\section{Conflict of Interest}

Puja Tanwani, Bruce A. Fernie, Ana V. Nikčević and Marcantonio M. Spada declared that they have no conflict of interest. 
American Psychiatric Association, 1994. Diagnostic and Statistical Manual of Mental Disorders: DSM-IV-TR®. American Psychiatric Pub.

Ávila, A., Cardona, X., Martín-Baranera, M., Bello, J., Sastre, F., 2011. Impulsive and compulsive behaviors in Parkinson's disease: A one-year follow-up study. Journal of the Neurological Sciences 310, 197-201.

Bermejo, P.E., 2008a. Topiramate in managing impulse control disorders in Parkinson's disease. Parkinsonism and Related Disorders 14, 448-449.

Bermejo, P.E., 2008b. Topiramate in managing impulse control disorders in Parkinson's disease. Parkinsonism and Related Disorders 14, 448-449.

Bortolato, M., Cannas, A., Solla, P., Bini, V., Puligheddu, M., Marrosu, F., 2012. Finasteride attenuates pathological gambling in patients with Parkinson disease. Journal of Clinical Psychopharmacology 32, 424-425.

Bosco, D., Plastino, M., Colica, C., Bosco, F., Arianna, S., Vecchio, A., Galati, F., Cristiano, D., Consoli, A., Consoli, D., 2012. Opioid antagonist naltrexone for the treatment of pathological gambling in Parkinson disease. Clinical Neuropharmacology 35, 118-120.

Chaudhuri, K., Schapira, A.H., 2009. Non-motor symptoms of Parkinson's disease: dopaminergic pathophysiology and treatment. The Lancet Neurology 8, 464-474.

Cilia, R., Siri, C., Marotta, G., Isaias, I.U., De Gaspari, D., Canesi, M., Pezzoli, G., Antonini, A., 2008. Functional abnormalities underlying pathological gambling in Parkinson disease. Archives of Neurology 65, 1604-1611.

Cormier, F., Muellner, J., Corvol, J.C., 2013. Genetics of impulse control disorders in Parkinson's disease. Journal of Neural Transmission 120, 665-671.

Delaney, M., Leroi, I., Simpson, J., Overton, P.G., 2012. Impulse control disorders in Parkinson's disease: a psychosocial perspective. Journal of Clinical Psychology in Medical Settings 19, 338-346. Demetriades, P., Rickards, H., Cavanna, A.E., 2011. Impulse control disorders following deep brain stimulation of the subthalamic nucleus in Parkinson's disease: clinical aspects. Parkinson's Disease 2011. 
Eisenegger, C., Knoch, D., Ebstein, R.P., Gianotti, L.R., Sándor, P.S., Fehr, E., 2010. Dopamine receptor D4 polymorphism predicts the effect of L-DOPA on gambling behavior. Biological Psychiatry 67, 702-706.

Emilio Bermejo, P., Ruiz-Huete, C., Anciones, B., 2010. Zonisamide in managing impulse control disorders in Parkinson's disease. Journal of Neurology 257, 1682-1685.

Evans, A.H., Katzenschlager, R., Paviour, D., O'Sullivan, J.D., Appel, S., Lawrence, A.D., Lees, A.J., 2004. Punding in Parkinson's disease: its relation to the dopamine dysregulation syndrome. Movement Disorders 19, 397-405.

Ferris, J.A., Stirpe, T., Ialomiteanu, A., 1996. Gambling in Ontario: a report from a general population survey on gambling-related problems and opinions. Addiction Research Foundation, Problem and Compulsive Gambling Project.

Friedman, J.H., 1994. Punding on levodopa. Biological Psychiatry 36, 350-351.

Giovannoni, G., O'sullivan, J., Turner, K., Manson, A., Lees, A., 2000. Hedonistic homeostatic dysregulation in patients with Parkinson's disease on dopamine replacement therapies. Journal of Neurology, Neurosurgery \& Psychiatry 68, 423-428.

Goodman, W.K., Price, L.H., Rasmussen, S.A., Mazure, C., Fleischmann, R.L., Hill, C.L., Heninger, G.R., Charney, D.S., 1989. The Yale-Brown obsessive compulsive scale: I. Development, use, and reliability. Archives of General Psychiatry 46, 1006-1011.

Guy, W., 1976. Clinical global impression scale. The ECDEU Assessment Manual for Psychopharmacology-Revised. Volume DHEW Publ No ADM 76 338, 218-222.

Halbig, T.D., Tse, W., Frisina, P.G., Baker, B.R., Hollander, E., Shapiro, H., Tagliati, M., Koller, W.C., Olanow, C.W., 2009. Subthalamic deep brain stimulation and impulse control in Parkinson's disease. European Journal of Neurology 16, 493-497.

Hardwick, A., Ward, H., Hassan, A., Romrell, J., Okun, M.S., 2013. Clozapine as a potential treatment for refractory impulsive, compulsive, and punding behaviors in Parkinson's disease. Neurocase 19, 587-591.

Kim, S.W., Grant, J.E., Potenza, M.N., Blanco, C., Hollander, E., 2009. The Gambling Symptom Assessment Scale (G-SAS): a reliability and validity study. Psychiatry Research 166, 76-84. 
Kurlan, R., 2004. Disabling repetitive behaviors in Parkinson's disease. Movement Disorders 19, 433437.

Leroi, I., Ahearn, D.J., Andrews, M., McDonald, K.R., Byrne, E.J., Burns, A., 2011. Behavioural disorders, disability and quality of life in Parkinson's disease. Age \& Ageing 40, 614-621. Limotai, N., Oyama, G., Go, C., Bernal, O., Ong, T., Moum, S.J., Bhidayasiri, R., Foote, K.D., Bowers, D., Ward, H., 2012. Addiction-like manifestations and Parkinson's disease: a large single center 9-year experience. International Journal of Neuroscience 122, 145-153.

Luginger, E., Wenning, G., Bösch, S., Poewe, W., 2000. Beneficial effects of amantadine on L-dopainduced dyskinesias in Parkinson's disease. Movement Disorders 15, 873-878. Mamikonyan, E., Siderowf, A.D., Duda, J.E., Potenza, M.N., Horn, S., Stern, M.B., Weintraub, D., 2008. Long-term follow-up of impulse control disorders in Parkinson's disease. Movement Disorders 23, 75-80.

Nikitina, M., Zhukova, I., Izhboldina, O., Zhukova, N., Alifirova, V., Agasheva, A., Povalyaev, A., Titova, M., 2013. Influence of impulse control disorders on the quality of life among cognitivelyintact Parkinson's disease patients living in the Tomsk region, Russia. Journal of Parkinson's Disease 3, 100-101.

Okai, D., Askey-Jones, S., Samuel, M., O'Sullivan, S.S., Chaudhuri, K., Martin, A., Mack, J., Brown, R.G., David, A.S., 2013. Trial of CBT for impulse control behaviors affecting Parkinson patients and their caregivers. Neurology 80, 792-799.

Patton, J.H., Stanford, M.S., 1995. Factor structure of the Barratt impulsiveness scale. Journal of Clinical Psychology 51, 768-774.

Phillips, B., Ball, C., Sackett, D., Badenoch, D., Straus, S., Haynes, B., Oxford Centre for Evidencebased Medicine levels of evidence (March 2009). Centre for Evidence Based Medicine Web site. Politis, M., Piccini, P., Pavese, N., Koh, S.-B., Brooks, D.J., 2008. Evidence of dopamine dysfunction in the hypothalamus of patients with Parkinson's disease: An in vivo 11C-raclopride PET study. Experimental Neurology 214, 112-116. 
Pondal, M., Marras, C., Miyasaki, J., Moro, E., Armstrong, M.J., Strafella, A.P., Shah, B.B., Fox, S., Prashanth, L., Phielipp, N., 2013. Clinical features of dopamine agonist withdrawal syndrome in a movement disorders clinic. Journal of Neurology, Neurosurgery \& Psychiatry 84, 130-135.

Pontone, G., Williams, J.R., Bassett, S.S., Marsh, L., 2006. Clinical features associated with impulse control disorders in Parkinson disease. Neurology 67, 1258-1261.

Rabinak, C.A., Nirenberg, M.J., 2010. Dopamine agonist withdrawal syndrome in Parkinson disease. Archives of Neurology 67, 58-63.

Rahman, S., Griffin, H.J., Quinn, N.P., Jahanshahi, M., 2008. Quality of life in Parkinson's disease: the relative importance of the symptoms. Movement Disorders 23, 1428-1434.

Reuter, J., Raedler, T., Rose, M., Hand, I., Gläscher, J., Büchel, C., 2005. Pathological gambling is linked to reduced activation of the mesolimbic reward system. Nature Neuroscience 8, 147-148. Sriram, A., Ward, H.E., Hassan, A., Iyer, S., Foote, K.D., Rodriguez, R.L., McFarland, N.R., Okun, M.S., 2013. Valproate as a treatment for dopamine dysregulation syndrome (DDS) in Parkinson's disease. Journal of Neurology 260, 521-527.

Stowe, R., Ives, N., Clarke, C., Van Hilten, J., Ferreira, J., Hawker, R., Shah, L., Wheatley, K., Gray, R., 2008. Dopamine agonist therapy in early Parkinson’s disease. Cochrane Database Syst Rev 2. Thomas, A., Bonanni, L., Gambi, F., Di Iorio, A., Onofrj, M., 2010. Pathological gambling in Parkinson disease is reduced by amantadine. Annals of Neurology 68, 400-404.

Voon, V., Hassan, K., Zurowski, M., Duff-Canning, S., De Souza, M., Fox, S., Lang, A., Miyasaki, J., 2006. Prospective prevalence of pathologic gambling and medication association in Parkinson disease. Neurology 66, 1750-1752.

Weintraub, D., 2009. Impulse control disorder: Prevalence and possible risk factors. European Neuropsychopharmacology 19, S196-S197.

Weintraub, D., Hoops, S., Shea, J.A., Lyons, K.E., Pahwa, R., Driver-Dunckley, E.D., Adler, C.H., Potenza, M.N., Miyasaki, J., Siderowf, A.D., Duda, J.E., Hurtig, H.I., Colcher, A., Horn, S.S., Stern, M.B., Voon, V., 2009a. Validation of the questionnaire for impulsive-compulsive disorders in Parkinson's disease. Movement Disorders 24, 1461-1467. 
Weintraub, D., Hoops, S., Shea, J.A., Lyons, K.E., Pahwa, R., Driver-Dunckley, E.D., Adler, C.H., Potenza, M.N., Miyasaki, J., Siderowf, A.D., 2009b. Validation of the questionnaire for impulsivecompulsive disorders in Parkinson's disease. Movement Disorders 24, 1461-1467.

Weintraub, D., Koester, J., Potenza, M.N., Siderowf, A.D., Stacy, M., Voon, V., Whetteckey, J., Wunderlich, G.R., Lang, A.E., 2010a. Impulse control disorders in Parkinson disease: a crosssectional study of 3090 patients. Archives of Neurology 67, 589-595.

Weintraub, D., Siderowf, A., 2012. Frequency of impulse control disorder symptoms in de novo Parkinson's disease patients and healthy controls. Movement Disorders 27, S291.

Weintraub, D., Siderowf, A.D., Potenza, M.N., Goveas, J., Morales, K.H., Duda, J.E., Moberg, P.J., Stern, M.B., 2006. Association of dopamine agonist use with impulse control disorders in Parkinson disease. Archives of Neurology 63, 969-973.

Weintraub, D., Sohr, M., Potenza, M.N., Siderowf, A.D., Stacy, M., Voon, V., Whetteckey, J., Wunderlich, G.R., Lang, A.E., 2010b. Amantadine use associated with impulse control disorders in Parkinson disease in cross-sectional study. Annals of Neurology 68, 963-968.

Weintraub, D., Xie, S.X., Stern, M., Hurtig, H., Siderowf, A., Minger, J., et al, 2013. Randomized, double-blind, placebo-controlled study of naltrexone for impulse control disorders in Parkinson's disease [abstract], Movement Disorders, p. 393.

Wood, L.D., Neumiller, J.J., Setter, S.M., Dobbins, E.K., 2010. Clinical review of treatment options for select nonmotor symptoms of Parkinson's disease. The American Journal of Geriatric Pharmacotherapy 8, 294-315. 
Table 1: Summary of studies retrieved of treatment for ICBs in patients with PD arranged according to type of study.

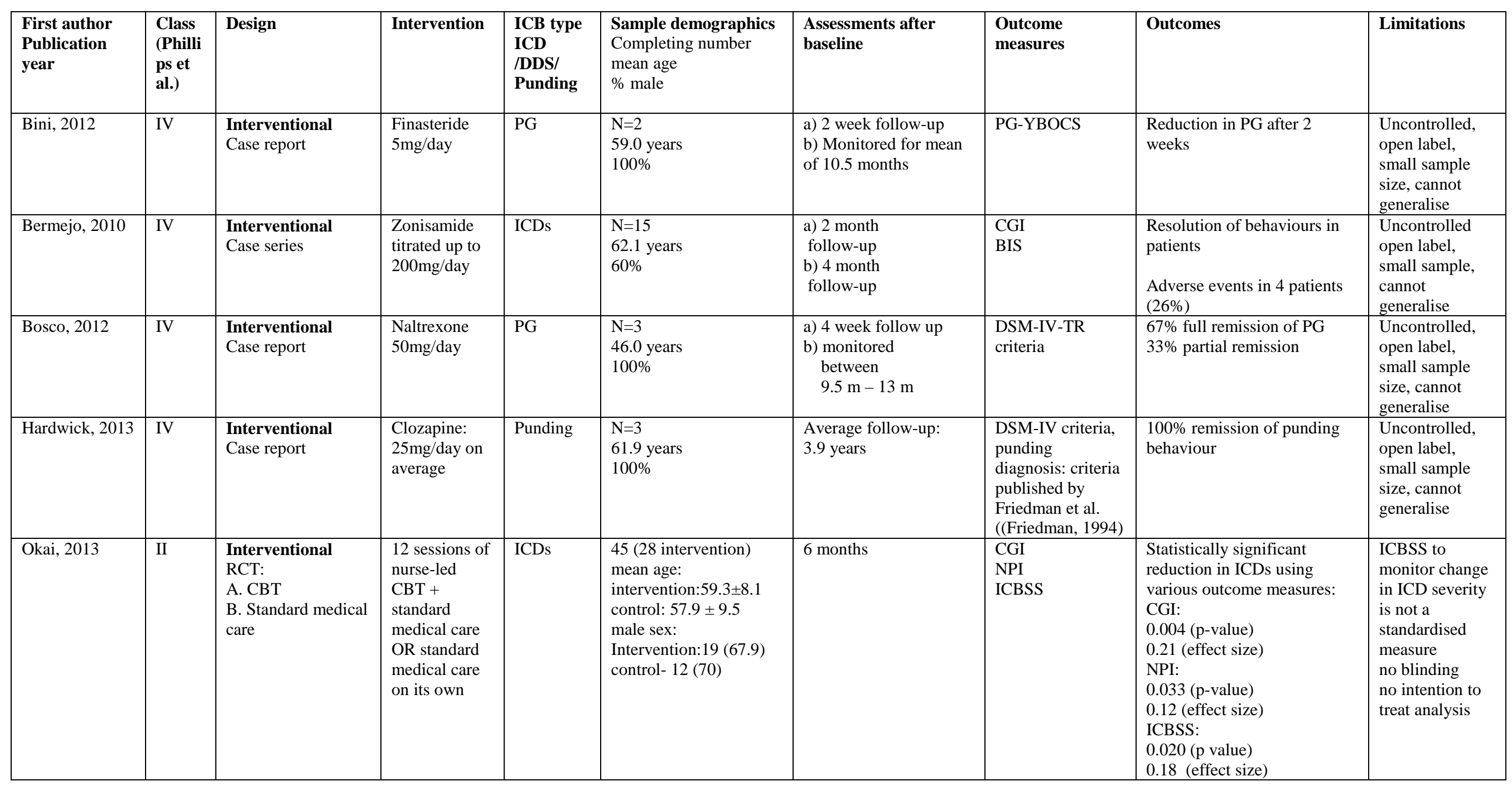




\begin{tabular}{|c|c|c|c|c|c|c|c|c|c|}
\hline Sriram, 2013 & IV & $\begin{array}{l}\text { Interventional } \\
\text { Case series }\end{array}$ & $\begin{array}{l}\text { Valproate } \\
\text { extended } \\
\text { release } \\
\text { (average } \\
562.5 \mathrm{mg} / \text { day) }\end{array}$ & DDS & $\begin{array}{l}\mathrm{N}=4 \\
62.3 \text { years } \\
50 \%\end{array}$ & $\begin{array}{l}\text { a) follow-up 3-6m } \\
\text { mean follow-up: } \\
5 \mathrm{~m}\end{array}$ & $\begin{array}{l}\text { Giovannoni } \\
\text { criteria for DDS } \\
\text { ((Giovannoni et } \\
\text { al., 2000) }\end{array}$ & $\begin{array}{l}\text { Symptoms resolved in all } \\
\text { patients }\end{array}$ & $\begin{array}{l}\text { Small number, } \\
\text { no standardised } \\
\text { measure used, } \\
3 / 4 \text { had DBS } \\
\text { which has been } \\
\text { implicated in } \\
\text { managing and } \\
\text { causing ICDs }\end{array}$ \\
\hline Tomas, 2013 & $\mathrm{I}$ & $\begin{array}{l}\text { Interventional } \\
\text { Double-blind } \\
\text { crossover RCT } \\
\text { A. amantadine } \\
\quad 200 \mathrm{mg} / \text { day } \\
\text { B. placebo } \\
\text { 17weeks }\end{array}$ & $\begin{array}{l}\text { Amantadine } \\
\text { 200mg/day }\end{array}$ & PG & $\begin{array}{l}\mathrm{N}=17 \text { (12 completed }) \\
61.0 \pm 1.6 \text { years } \\
76.5 \%\end{array}$ & a) 4 week follow-up & $\begin{array}{l}\text { DSM-IV criteria } \\
\text { G-SAS } \\
\text { PG-YBOCS } \\
\text { +daily diary } \\
\text { entries }\end{array}$ & $\begin{array}{l}\text { Statistically significant } \\
\text { reduction in all outcome } \\
\text { measures: } \\
\text { G-SAS and PG-YBOCS } \\
(p<0.001) \\
5 \text { patients dropped out due to } \\
\text { adverse events (hallucinations } \\
\text { and confusion) }\end{array}$ & $\begin{array}{l}\text { Short study } \\
\text { duration, } \\
\text { small sample } \\
\text { size }\end{array}$ \\
\hline
\end{tabular}

Abbreviations: ICDs: Impulsive-Compulsive Disorders; DDS: Dopamine Dysregulation Syndrome; PG: Pathological Gambling; RCT: Randomised-Control Trial; CBT: Cognitive-Behavioural Therapy; PRS: Punding Rating Scale; DSM-IV: Diagnostic and Statistical Manual of Mental Disorders: Fourth Edition; CGI: Clinical Global Impression; NPI: Neuropsychiatric Inventory; ICBSS: Impulse Control Behaviour Severity Scale; MMSE: Mini-Mental State Examination; G-SAS: Gambling Symptom Assessment Scale; PG-YBOCS: Yale-Brown Obsessive Compulsive Scale modified for Pathological Gambling. 